Received: 11 January 2017

Accepted: 9 October 2017

Published online: 27 October 2017
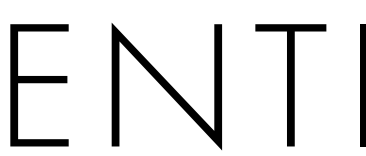

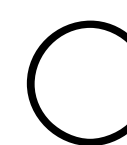

REP

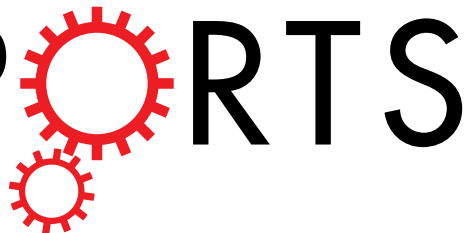

Amended: Addendum

\title{
OPEN Exercise-dependent formation of new junctions that promote STIM1- Orai1 assembly in skeletal muscle
}

\author{
Simona Boncompagni ${ }^{1,2}$, Antonio Michelucci ${ }^{1,2}$, Laura Pietrangelo ${ }^{1,2}$, Robert T. Dirksen ${ }^{3}$ \& \\ Feliciano Protasi ${ }^{1,4}$
}

Store-operated $\mathrm{Ca}^{2+}$ entry (SOCE), a ubiquitous mechanism that allows recovery of $\mathrm{Ca}^{2+}$ ions from the extracellular space, has been proposed to limit fatigue during repetitive skeletal muscle activity. However, the subcellular location for SOCE in muscle fibers has not been unequivocally identified. Here we show that exercise drives a significant remodeling of the sarcotubular system to form previously unidentified junctions between the sarcoplasmic reticulum (SR) and transverse-tubules (TTs). We also demonstrate that these new SR-TT junctions contain the molecular machinery that mediate SOCE: stromal interaction molecule-1 (STIM1), which functions as the $\mathrm{SR} \mathrm{Ca}^{2+}$ sensor, and Orai1, the $\mathrm{Ca}^{2+}$. permeable channel in the TT. In addition, EDL muscles isolated from exercised mice exhibit an increased capability of maintaining contractile force during repetitive stimulation in the presence of $2.5 \mathrm{mM}$ extracellular $\mathrm{Ca}^{2+}$, compared to muscles from control mice. This functional difference is significantly reduced by either replacement of extracellular $\mathrm{Ca}^{2+}$ with $\mathrm{Mg}^{2+}$ or the addition of SOCE inhibitors (BTP-2 and 2-APB). We propose that the new SR-TT junctions formed during exercise, and that contain STIM1 and Orai1, function as $\mathrm{Ca}^{2+}$ Entry Units (CEUs), structures that provide a pathway to rapidly recover $\mathrm{Ca}^{2+}$ ions from the extracellular space during repetitive muscle activity.

Store-operated $\mathrm{Ca}^{2+}$ entry (SOCE) is a ubiquitous $\mathrm{Ca}^{2+}$ entry mechanism, first described in non-excitable cells, that is triggered by depletion of intracellular $\mathrm{Ca}^{2+}$ stores (endoplasmic/sarcoplasmic reticulum, respectively ER and SR) ${ }^{1,2}$. SOCE is coordinated by the communication between: a) stromal interaction molecule-1 (STIM1), which acts as the $\mathrm{Ca}^{2+}$ sensor in the ER lumen ${ }^{3,4,5}$, and b) Orail, the $\mathrm{Ca}^{2+}$-permeable channel in the plasma membrane $(\mathrm{PM})^{6,7}$. The mechanism for Orail activation in non-excitable cells involves store depletion causing STIM1 oligomers to aggregate and translocate to form junctions with the $\mathrm{PM}^{8-10}$. Aggregated STIM1, in turn, recruits and traps Orail channels into these ER-PM junctions, or puncta, resulting in the activation of Orail channels that mediate entry of $\mathrm{Ca}^{2+}$ into the cell from the extracellular space ${ }^{11}$.

SOCE in skeletal muscle ${ }^{12-14}$ is similarly mediated by interactions between STIM1 in the SR and Orail channels in the $\mathrm{PM}^{15,16}$ and is proposed to limit muscle fatigue during repetitive stimulation ${ }^{17}$. Recently, using constitutive and inducible, muscle-specific Orail knockout mice, Carrell and collegues reported that while Orail limits muscle fatigue by promoting the growth and maintenance of fatigue-resistant type I fibers, fatigue and endurance exercise were unaltered following post-developmental, muscle-specific ablation of Orai $1^{18}$. Thus, the precise mechanisms by which Orail impacts muscle fatigue remain controversial. In addition to its role in healthy muscle, altered SOCE activity is also linked to several distinct forms of muscle dysfunction. For example, a reduction in SOCE activity was proposed to contribute to muscle impairment in aging ${ }^{19,20}$, though this finding was not confirmed by others ${ }^{21,22}$. On the other hand, an increase in Orail-mediated SOCE exacerbates muscular dystrophy in $m d x$ mice ${ }^{23-26}$ and mutations in STIM1 and Orail are linked to Tubular Aggregate Myopathy (TAM) $)^{27-31}$, an autosomal dominant muscle disease that is clinically characterized by myalgia, cramps and muscle stiffness, with or without proximal muscle weakness. Together, these findings from prior studies suggest that Orail-mediated SOCE plays an important role in skeletal muscle function and disease.

${ }^{1} \mathrm{CeSI}$-Met - Center for Research on Ageing and Translational Medicine, University G. d'Annunzio, Chieti, I-66100, Italy. ${ }^{2}$ DNICS - Department of Neuroscience, Imaging and Clinical Sciences, University G. d'Annunzio, Chieti, I-66100, Italy. ${ }^{3}$ Department of Pharmacology and Physiology, University of Rochester Medical Center, Rochester, NY, 14642, USA. ${ }^{4}$ DMSI - Department of Medicine and Aging Science, University G. d'Annunzio, Chieti, I-66100, Italy. Simona Boncompagni and Antonio Michelucci contributed equally. Correspondence and requests for materials should be addressed to F.P. (email: feliciano.protasi@unich.it) 
Experiments in skinned skeletal muscle fibers have provided evidence that SOCE channels are present in transverse tubules (TTs), specialized invaginations of the PM that propagate the action potential into the fiber interior, and activate rapidly following SR Ca ${ }^{2+}$ store depletion ${ }^{12,32}$. These studies suggest that rapid SOCE in the TT system might involve STIM1-Orail coupling within the pre-formed SR-TT junctions of the triad, sites of excitation-contraction (EC) coupling also known as $\mathrm{Ca}^{2+}$ release units (CRUs) ${ }^{33,34}$. However, to date the precise subcellular sites of STIM1-Orail interaction during SOCE in skeletal muscle fibers has not been unequivocally identified. Indeed, given the tight packing of type 1 ryanodine receptor (RyR1) arrays in the terminal $\mathrm{SR}^{33}$, there may not be sufficient space within the triad to also accommodate large STIM1 aggregates needed to trap and activate Orail channels following store depletion. In addition, the junctional gap width of the triad $(\sim 12 \mathrm{~nm})$ is larger than the corresponding gap width $(\sim 8 \mathrm{~nm})$ between the ER and PM that accommodates STIM1 aggregates as estimated in non-excitable cells transfected with YFP-STIM $1^{35}$.

Here we used a combination of electron microscopy (EM), immunofluorescence, immunogold, and ex vivo muscle contractility techniques to assess STIM1 and Orail subcellular localization and skeletal muscle function in adult mice under resting conditions and after a single bout of treadmill exercise. Our results show that exercise drives a significant remodeling of the sarcotubular system within the I band of the sarcomere that results in the formation of previously unidentified junctions between the SR and TTs that contain STIM1 and Orail proteins. In addition, this remodeling is accompanied by an increased resistance to fatigue during repetitive high frequency stimulation that is significantly reduced by preventing $\mathrm{Ca}^{2+}$ influx following either removal of extracellular $\mathrm{Ca}^{2+}$ or addition of SOCE inhibitors.

\section{Results \\ Treadmill exercise drives rearrangement of membranes at the I band into stacks of flat-cister-}

nae. In mammalian skeletal muscle, the SR is composed of two morphologically and functionally different regions: 1) the junctional SR (also known as SR terminal cisternae) located at the I-A band transition of relaxed sarcomeres, which associates with TTs to form triads (labeled SR-TT-SR in Fig. 1A,C); and 2) the free-SR, which is localized either next to the A band or within the I band (on both sides of Z lines). In EM, the free-SR at the I band is formed by convoluted tubules that appear as multiple layers of vesicles when viewed in cross-sections (Fig. 1B). However, following a single session of treadmill exercise ( $\sim$ hour while progressively increasing treadmill speed from $10 \mathrm{~m} / \mathrm{min}$ to $25 \mathrm{~m} / \mathrm{min}$ ), regions of the free-SR within the I band exhibited a profound remodeling that involved the formation of stacks of flat-cisternae composed of multiple elements (Fig. 1C,D, empty arrows). In Fig. S1, the positioning of these stack of flat-cisternae at the I band (between the triad and the $\mathrm{Z}$ line) was more evident. In addition to SR, these stacks also contained TT membranes (see next paragraph). Stacks of flat-cisternae were also occasionally observed in muscle from non-exercised mice, but were significantly more frequent (Fig. 1E,F), larger in size, and formed by more elements (Fig. S2) following treadmill exercise. Table 1 summarizes the number/area of EM section of stacks of flat-cisternae to that of CRUs (i.e. triads) in EDL muscles from control and exercised mice. Under control conditions, EDL muscles contained $73.6 \pm 2.1 \mathrm{CRUs} / 100 \mu \mathrm{m}^{2}$ and only $2.0 \pm 0.3 / 100 \mu \mathrm{m}^{2}$ stacks of flat cisternae (ratio of 37:1; Table 1, column C). However, following exercise, the number of membrane stacks increased significantly (Fig. 1E,F; and Table 1, column B), while the number of CRUs remained constant (Table 1, column A). As a result, the ratio between CRUs and stacks of flat-cisternae decreased from 37:1 at rest to 7:1 following exercise (Table 1, column C). Finally, we quantified two additional parameters:

a. Small electron-dense strands were present between SR vesicles in control (inset in Fig. 1B) and between flat cisternae following exercise (inset in Fig. 1D). We measured the junctional gap size containing electron-dense strands and compared it with the junctional gap occupied by RyR1-feet in triads (as shown in Fig. 1G, top). The gap size in control samples (measured between SR vesicles where small electron-dense strands were visible) was significantly smaller than that of triads: $8.4 \pm 0.1 \mathrm{vs.} 12.7 \pm 0.3 \mathrm{~nm}$ $(\mathrm{p}<0.01)$ (Fig. 1G, bottom). Following exercise, while the junctional gap size in triads remained unaltered $(12.9 \pm 0.3 \mathrm{~nm})$, the gap size in stacks of flat-cisternae (containing small electron-dense strands) was significantly reduced from $8.4 \pm 0.1$ to $7.4 \pm 0.1 \mathrm{~nm}(\mathrm{p}<0.01)$.

b. Following exercise, membrane vesicles located near stacks of flat-cisternae (those marked by asterisks in Fig. 1D) were significantly larger than those observed in control muscles, a visual observation supported by direct measurements from EM images (Fig. 1H).

Following exercise, TT extensions at the I band are significantly more frequent and are associated with stacks of flat-cisternae. We stained the TT network with ferrocyanide, a precipitate that enters the TT lumen from the extracellular space to enable direct visualization of TTs by EM (Fig. 2; and Fig. S3). While in control muscles, longitudinal extensions of the TT were occasionally observed in the I band (Fig. S3A,C and D), they were much more frequent following treadmill exercise (Fig. S3B,E and F). These TTs: a) extended longitudinally/obliquely into the I band region, while maintaining continuity with the transversal portion of the TT that constitutes the central element of the triad (Fig. 2A-D); and b) they were often seen associated with stacks of flat-cisternae (Fig. 2,D; inset in Fig. S3F). A direct continuity between a triadic TT profile and it's I band extension associated within a stack of flat-cisternae is clearly visible in Fig. 2E. The increase of TT extensions into the I band following exercise was confirmed in EM cross sections, which enabled direct measurement of the total TT extension $/ 100 \mu \mathrm{m}^{2}$ (Fig. $2 \mathrm{~F}$ ).

Exercise promotes STIM1-Orai1 co-localization at the I band. To determine the subcellular localization of STIM1 and Orail under control conditions and following treadmill exercise, small bundles of EDL fibers 


\section{Control}
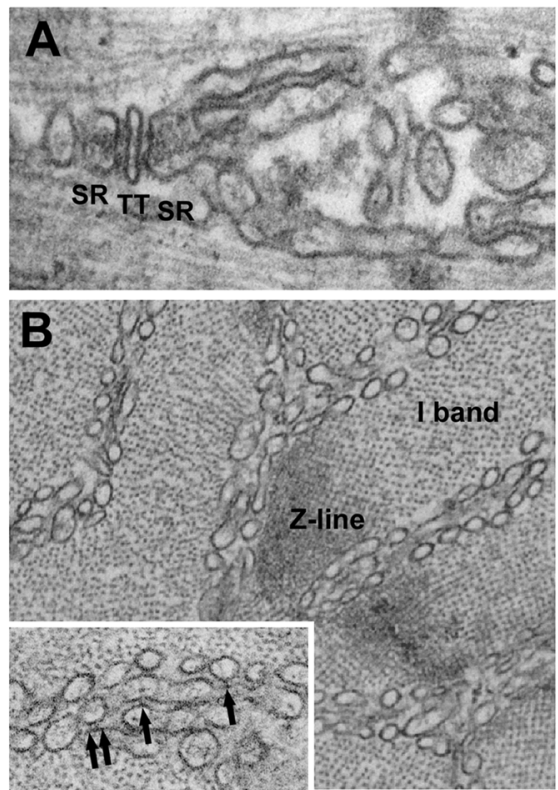

$\mathbf{E}$

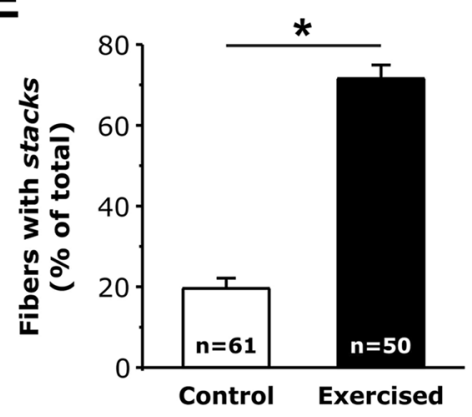

\section{Exercised}
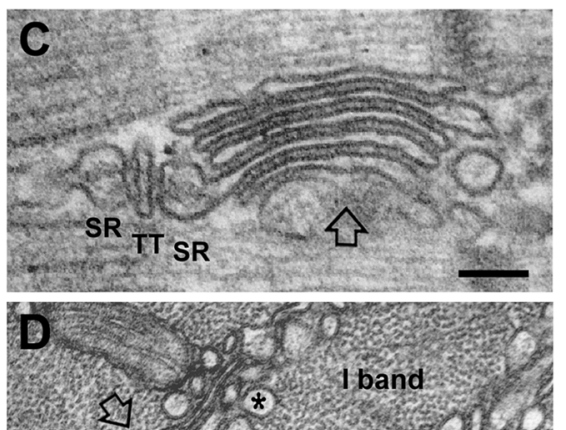
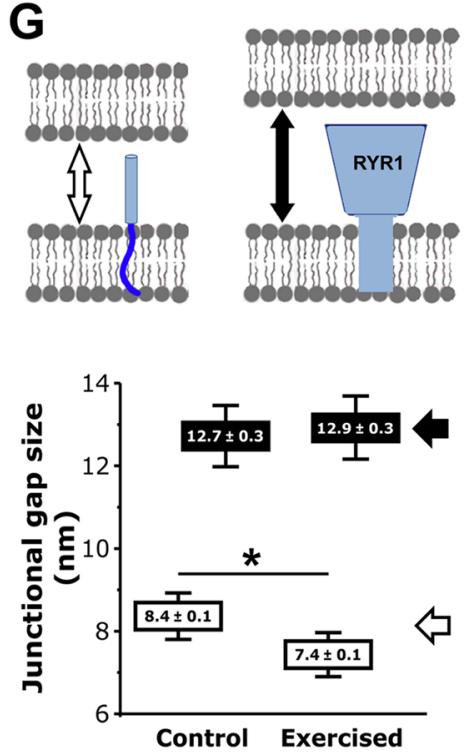

Figure 1. Following exercise, membranes at the I band rearrange into stacks of flat-cisternae. (A-D)

Longitudinal (A and $\mathbf{C}$ ) and cross (B and $\mathbf{D})$ EM sections in proximity of the I bands. Empty arrows in ( $\mathbf{C}$ and D) point to newly formed stacks of flat-cisternae; arrows in insets point to strands between SR vesicles (B) and flat-cisternae (D). (E and F) Incidence of stacks. Sample size: control, 3 mice; exercised, 3 mice. (G) Junctional gap size in membrane stacks (empty arrows) and in triads (black arrows) measured as shown in the cartoon. Samples size: control, 3 mice/76 measurements/21 stacks analyzed; exercised, 3 mice/119 measurements/34 stacks analyzed. (H) Average size of SR vesicles at the I band (some larger ones are marked by asterisks in panel D). Sample size: control, 3 mice/561 measurements; exercised, 3 mice/309 measurements. Data are shown as mean \pm SEM; ${ }^{*} \mathrm{p}<0.01$. Numbers in bars $(\mathrm{n})$ indicate the number of fibers analyzed $(\mathbf{E}$ and $\mathbf{F})$ and the number of SR vesicles measured (H). Scale bars: (A and C) (and insets in $\mathbf{B}$ and $\mathbf{D}$ ), $0.1 \mu \mathrm{m}$; (B) and (D), $0.2 \mu \mathrm{m}$.

\begin{tabular}{|l|l|l|l|}
\hline & A & B & C \\
\cline { 2 - 4 } & No. of CRUs/100 $\boldsymbol{\mu m}^{\mathbf{2}}$ & No. of stacks/100 $\boldsymbol{\mu \mathbf { m } ^ { \mathbf { 2 } }}$ & CRUs/stacks ratio \\
\hline Control & $73.6 \pm 2.1$ & $2.0 \pm 0.3$ & $37 / 1$ \\
\hline Exercised & $71.9 \pm 1.8$ & $9.9 \pm 0.7^{*}$ & $7 / 1$ \\
\hline
\end{tabular}

Table 1. The number/area of stacks of flat-cisternae, but not that of triads, increases following exercise. Columns A and B) number/area of CRUs and of stacks of flat- cisternae. Column C) Ratio between number/ area of CRUs and stacks of flat-cisternae. Sample size: control, 2 mice (14 fibers and 5-10 micrographs/fiber); exercised, 3 mice (21 fibers and 5 micrographs/fiber). Data are shown as mean $\pm \mathrm{SEM} ; \mathrm{p}<0.01$ vs. control.

were double-labeled in immunofluorescence experiments (Fig. 3) as follow: RyR1 vs. STIM1 (Fig. 3A,D), RyR1 vs. Orail (Fig. 3B,E), and STIM1 vs. Orail (Fig. 3C,F).

The staining of RyR1 was used as a known reference point marking the position of CRUs (i.e. triads): in adult fibers, triads were located at the A-I band junction on both sides of $Z$ lines to form a double set of transverse cross striations, marked as RyR1-positive puncta (red signal in Fig. 3A,D). In resting muscle, STIM1 was localized throughout the entire I band region, with peaks of the green STIM1 signal of variable intensity (Fig. 3A). 


\section{Exercised}
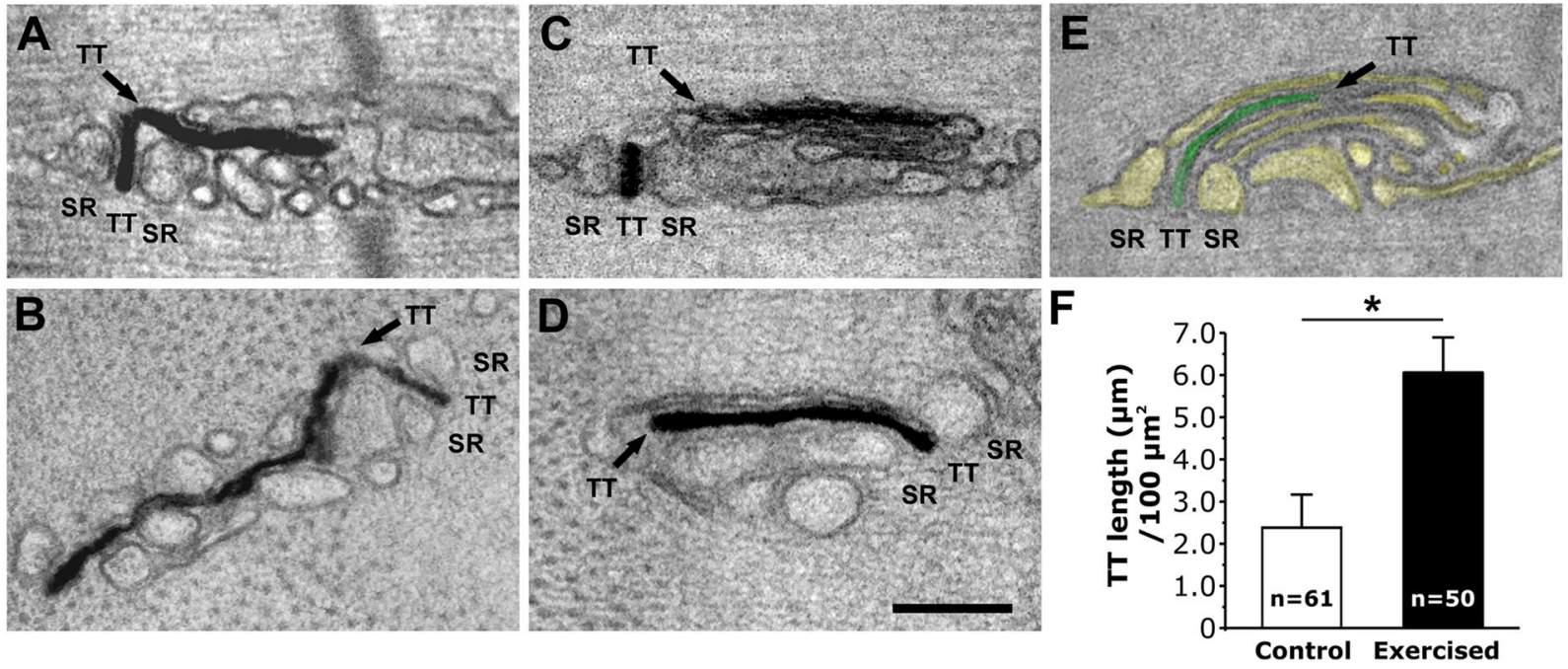

Figure 2. Following exercise, TT extensions at the I band are more frequent and become part of stacks of flatcisternae. (A-D) Representative EM images (A and C, longitudinal; B and D, cross) showing TTs (stained black with ferrocyanide-precipitate) extending from the triad into the I band to make contact with SR vesicles (A and $\mathbf{B}$ ) and stacks of flat-cisternae (C and D). (E) TT and SR (green and yellow, respectively) extending from a triad to form a lateral stack of flat-cisternae. (F) Quantitative analysis of the TT network extension at the I band measured in cross sections (see also Fig. S3C-F). Sample size: control, 3 mice; exercised, 3 mice. Data are shown as mean \pm SEM; ${ }^{*} \mathrm{p}<0.01$. Numbers in bars $(\mathrm{n})$ indicate the number of fibers analyzed. Scale bar: $0.1 \mu \mathrm{m}$.

In muscles from exercised mice, STIM1 retained the same location at the I band, though exhibiting spots that appeared brighter and more organized as puncta than in control samples (compare insets in Fig. 3A,D). The low presence of yellow color, due to limited overlap between STIM1 and RyR1 fluorescence, and low Pearson's correlation coefficient value are indicative of minimal STIM1-RyR1 co-localization under both control and exercised conditions (Fig. 3A,D). In contrast to STIM1, Orail labeling of control fibers resulted in significant co-localization with RyR1, as indicated by overlapping of peaks of RyR1 and Orail fluorescence, significant yellow signal in the merged image, and a relatively high Pearson's correlation coefficient value (Fig. 3B). This labeling is consistent with a preferential localization of Orail in TTs at the triad in control samples. As a consequence of a high RyR1-Orail co-localization, the level of Orail co-localization with STIM1 at rest is low, as indicated by the relatively low Pearson's correlation coefficient value (Fig. 3C).

While RyR1 and STIM1 positioning did not change following exercise (Fig. 3D-F), a fraction of the Orail signal shifted toward the I band in muscles from exercised mice (Fig. 3E and Fig. S3), resulting in a significant reduction of RyR1-Orail co-localization (compare Pearson's correlation coefficient values in Fig. 3B,E; p < 0.01). As a consequence of this shift in Orail location, STIM1-Orail co-localization was significantly increased, as shown by superimposition of peaks of fluorescence (traces in Fig. 3F), increased yellow signal in the merged image (inset in Fig. 3F), and an increased Pearson's correlation coefficient value (compare Pearson's correlation coefficient values in Fig. 3C,F; $\mathrm{p}<0.01$ ). The increased presence of Orail at the I band is consistent with the elongation of Orail-containing TT extensions toward the Z-line (see Fig. 2; and Fig. S3).

Immunogold labeling confirms an increased presence of Orai1 in the I band following exercise. To better define the localization of STIM1 and Orail at the ultra-structural level, we performed immunogold (IG) labeling in muscles from control and exercised mice (Fig. 4). RyR1, STIM1, and Orail IG labeling in EM was then analyzed by generating histograms for the distance of each gold particle from the nearest Z-line (Fig. 4). As for immunofluorescence (Fig. 3), we used RyR1 antibodies to label the position of CRUs/triads, and marked their position with respect to the Z-line with a cyan bar in each of the histograms. The results in Fig. 4 indicate that:

a. The position of both RyR1 and STIM1 did not change significantly following exercise: RyR1 remained at the triad and STIM1 remained preferentially present throughout the I band (Fig. 4-top and -center respectively; see also Fig. S4), even though some overlap between STIM1 and RyR1 was observed.

b. Following exercise, the Orail signal shifted from the triad junction toward the I band (arrow in Fig. 4-bottom; see also Fig. S4), where remodeling of the sarcotubular system led to the formation of stacks of flat-cisternae (Figs 1 and S1). Note that these stacks contained electron-dense strands (Fig. S5A and B) that were specifically labeled by STIM1-immunogold particles (Fig. S5C and D).

The brackets with numeric-values above each histogram in Fig. 4 show the percentage of gold particles that fell within the triad-area vs. those that fell within the I-band area for each of the 3 proteins analyzed (RyR1, STIM1, and Orai1). Importantly, these analyses showed that, following exercise, while STIM1 levels increased both at the 


\section{Control}
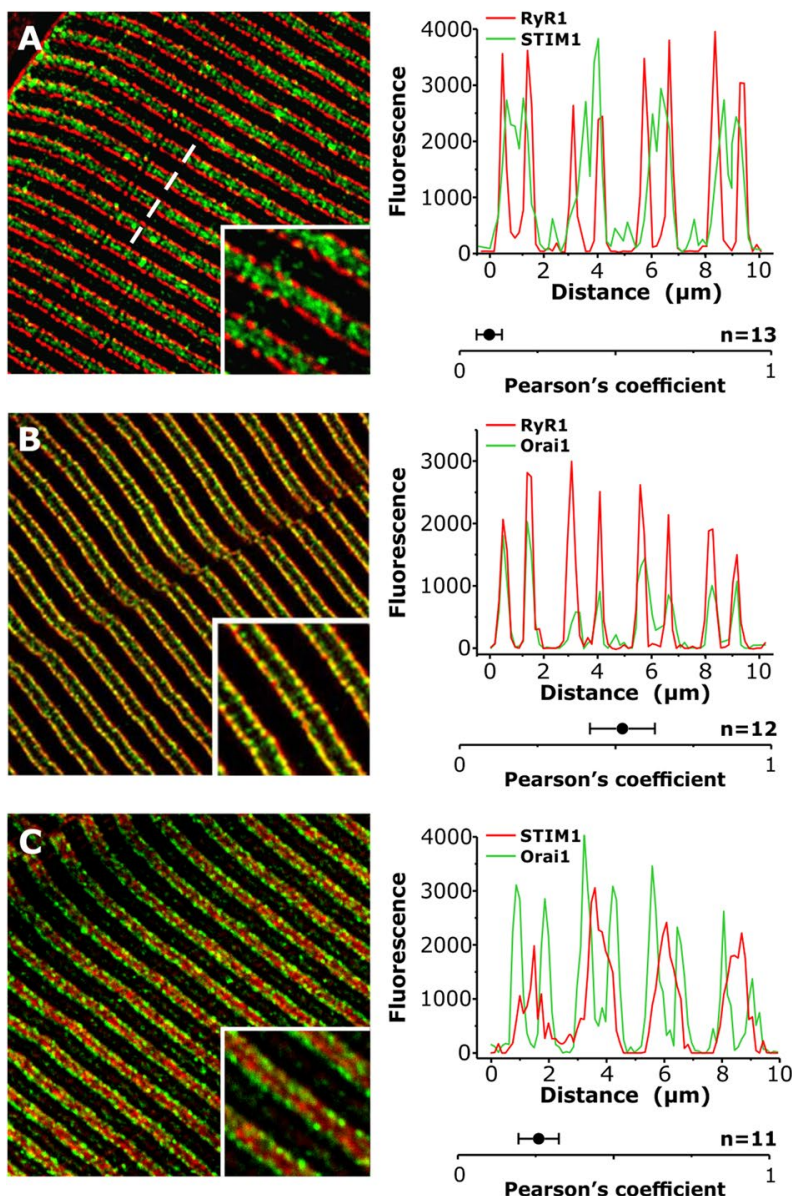

\section{Exercised}
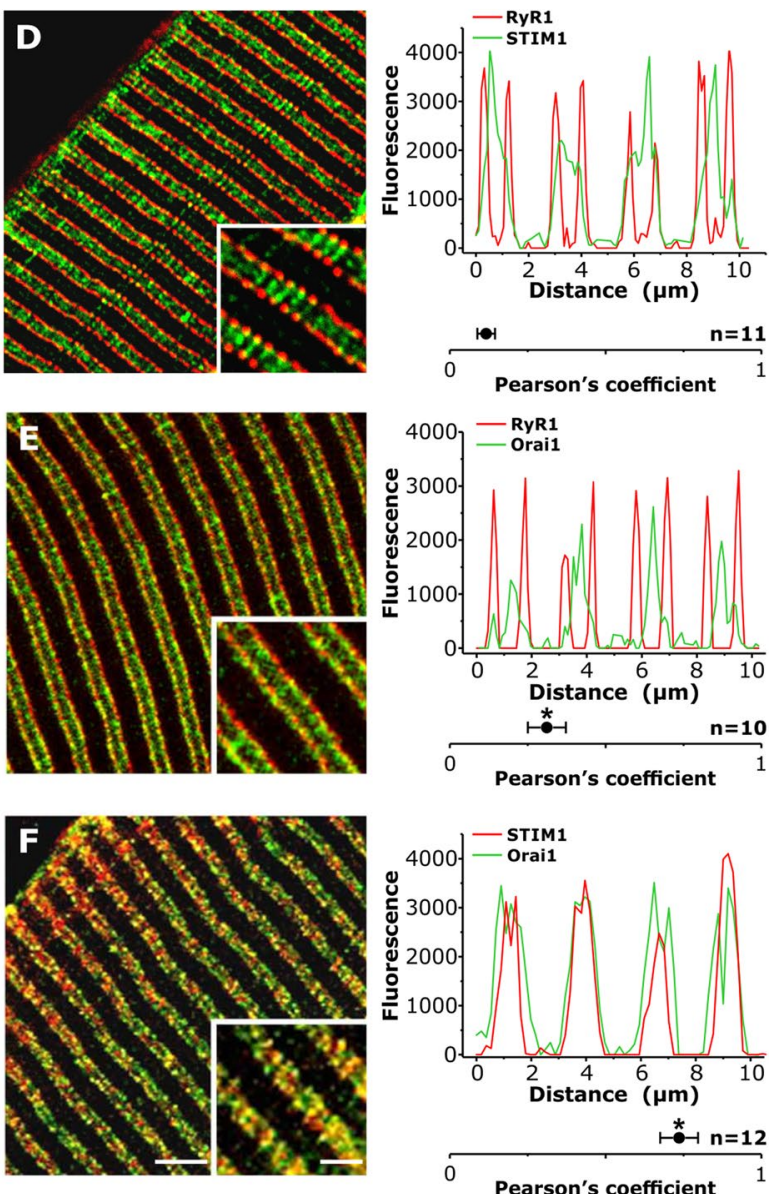

Figure 3. Co-localization between STIM1 and Orai1, low in control samples, increases significantly following exercise. Representative immunofluorescence images obtained from EDL fibers double-labeled for RyR1STIM1 (A and D), RyR1-Orail (B and E), and STIM1-Orail (C and F). Each panel also contains a fluorescence intensity profile along 4 sarcomeres (see dashed line in $\mathbf{A}$ ) and the Pearson's correlation coefficient value, a measure of covariance of pixel intensities, given as the mean \pm SEM; $* p<0.01$, compared to fibers from control mice. Numbers in figure (n) indicate the number of images analyzed. Scale bar: $2.5 \mu \mathrm{m}$ (insets: $1 \mu \mathrm{m}$ ).

triad (from 20\% to 33\%) and I band (38\% to 49\%), Orail levels decreased in the triad (56\% to 44\%) and increased in the I band (between 100 and $350 \mathrm{~nm}$ from the Z-line; 29 to 48\%). Additionally, non-linear curve fit analyses of the distribution of immunogold particle labeling for RyR1, STIM1, and Orail in EDL muscles from control (A) and exercised (B) mice confirmed a shift from the triad toward the I-band for only Orail (Fig. S4).

EDL muscles from exercised mice exhibit enhanced resistance to repetitive high frequency fatigue when $\mathrm{Ca}^{2+}$ entry is not prevented. We next compared the resistance to fatigue of EDL muscles excised from control and exercised mice. Before starting the fatigue protocol, muscles were first subjected to a standard force-frequency stimulation protocol using stimulus trains from 1 to $250 \mathrm{~Hz}$. No difference was observed in either the peak specific force-frequency or normalized force-frequency curves for EDL muscles from control and exercised mice (Fig. S6A and B). However, EDL muscles from exercised mice exhibited an increased ability to maintain contractile force during a repetitive high frequency stimulation protocol (30 consecutive $1 \mathrm{~s}$ duration $60 \mathrm{~Hz}$ stimuli delivered every 5 seconds) compared to that of muscles from control mice (Fig. 5A,B; Fig. S7A and E). Specifically, the average fractional force during the $15^{\text {th }}$ stimulus train (Fig. 5A) was significantly greater in EDL muscles from exercised mice $(0.74 \pm 0.03)$ compared to that of EDL muscles from control mice $(0.46 \pm 0.03)$ (Fig. 5B). Similar results were observed when using a stimulation rate $(100 \mathrm{~Hz})$ at the peak of the force-frequency, though the magnitude of the increased ability to maintain contractile force was less than that observed at the lower frequency (Fig. S6C and D).

To assess the role of $\mathrm{Ca}^{2+}$ entry in the enhanced fatigue resistance of EDL muscles from exercised mice, parallel experiments were conducted using either a $\mathrm{Ca}^{2+}$-free $\mathrm{KH}$ solution in which $\mathrm{Ca}^{2+}$ was replaced with $\mathrm{Mg}^{2+}$ (Fig. 5C-F; and Fig. S1B and F) or a $\mathrm{Ca}^{2+}$-containing KH solution supplemented with either $10 \mu \mathrm{M} \mathrm{BTP}-2$ 

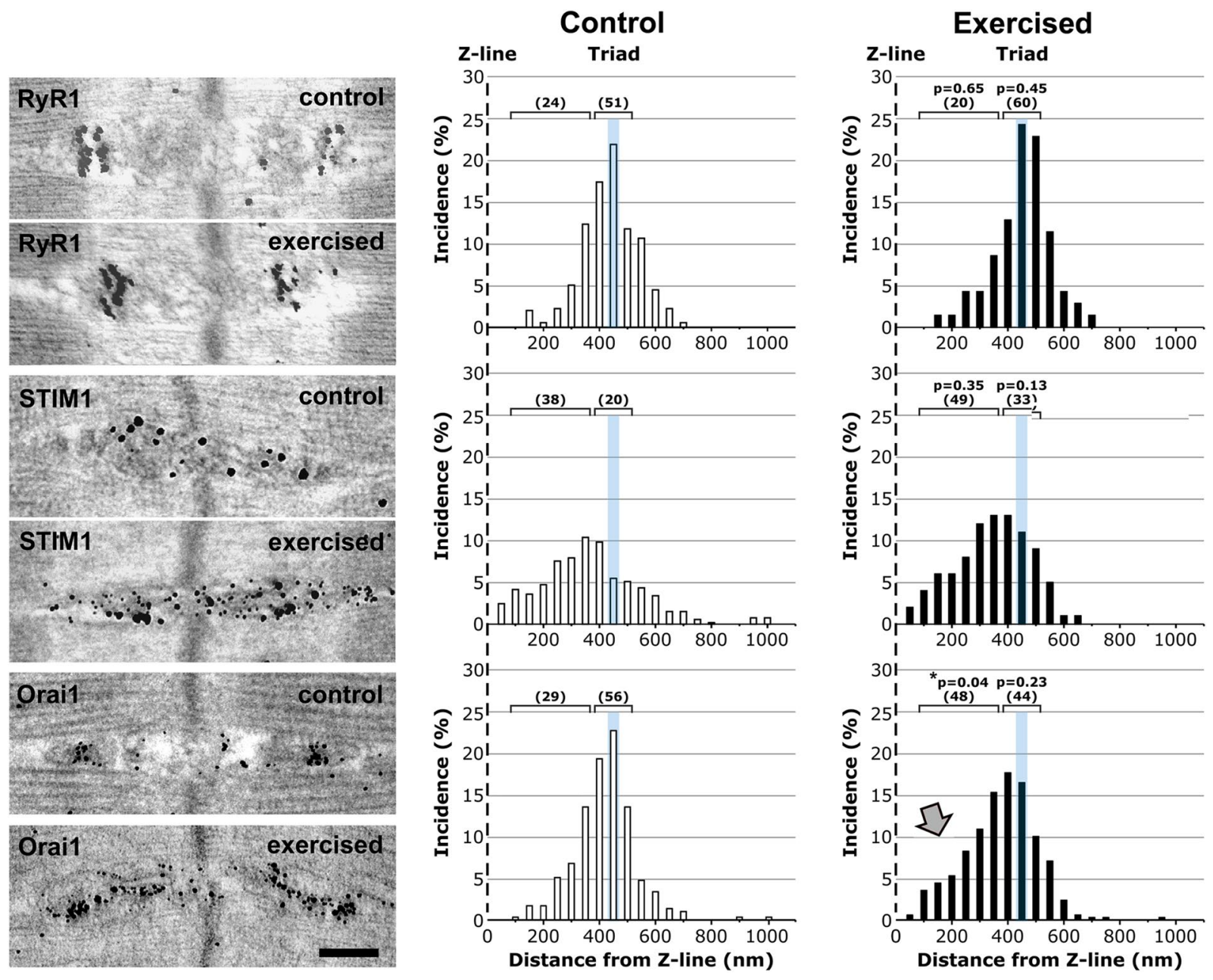

Figure 4. Immunogold labeling shows i) preferential I band localization of STIM1 and ii) increased presence of Orail at the I band following exercise. Left) Representative immunogold EM images showing the localization of RyR1 (top), STIM1 (center), and Orail (bottom) under control and exercised conditions. Right) Histograms of distances of immunogold particles from the $\mathrm{Z}$ line. The cyan line marks the position of the TT at the triad. Brackets with numeric values indicate the percentage of gold particles that fall within the triad-area vs. those that fall within the I-band area. Grey arrow points to the increased presence of Orail at the I-band following exercise. Sample size: control, 2 mice/4-8 fibers analyzed; exercised, 3 mice/3-8 fibers analyzed. *p $<0.05$. Scale bar: $0.2 \mu \mathrm{m}$.

(Fig. 5C-F; and Fig. S7C and G) or $100 \mu \mathrm{M}$ 2-APB (Fig. 5C-F; and Fig. S7D and H), established inhibitors of $\mathrm{SOCE}^{36-39}$. These studies revealed that the enhanced fatigue resistance of EDL muscles from exercised mice was no longer significantly different from controls in either the absence of extracellular $\mathrm{Ca}^{2+}$ or presence of SOCE inhibitors. Specifically, the significant increase in force during the $15^{\text {th }}$ stimulus train observed in EDL muscles from exercised mice was no longer observed under conditions that prevented SOCE (Fig. 5C-F).

\section{Discussion}

SOCE is a major pathway for $\mathrm{Ca}^{2+}$ influx from the extracellular space in skeletal muscle fibers. The importance of this process for muscle function is supported by prior studies demonstrating a potential role for SOCE in muscle fatigue $^{17,18,40}$, ageing ${ }^{19,20,41}$, and myopathy ${ }^{23,24,27-29}$. In spite of these advances, the precise subcellular localization of proteins involved in SOCE (STIM1 and Orai1) in skeletal muscle fibers has not been unequivocally identified.

Main findings. Our results indicate that: a) Exercise drives a remodeling of the existing sarcotubular system within the I band of the sarcomere that leads to the formation of stacks of membranes (Fig. 1 and Fig. S1) containing two distinct components: TT extensions into the I band (Fig. 2; and Fig. S3) and stacks of flat SR cisternae. b) The newly formed SR-TT junctions promote increased co-localization of STIM1 and Orail at the I band (Figs 3 and 4; and Fig. S4). While the frequency of new TT-SR junctions within the I band (i.e. distinct from triads) increases following treadmill exercise, these junctions are also present at a lower frequency and smaller in size in resting muscles (Fig. 1, Fig. S2, and Table 1), and thus, may contribute to sites of STIM1-Orai1 proximity under control conditions. c) EDL muscles isolated from exercised mice are more resistant to fatigue than EDL muscles 
A

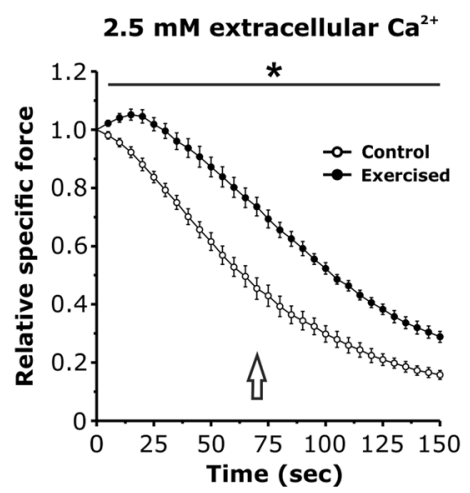

B

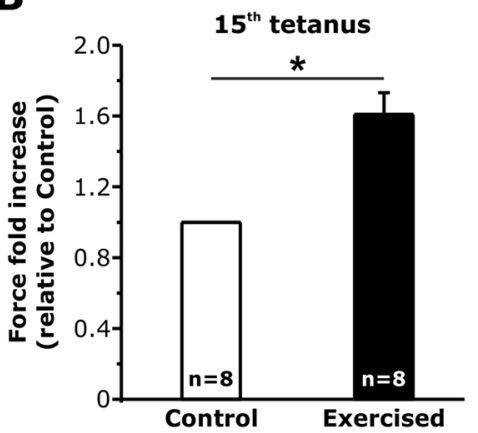

C

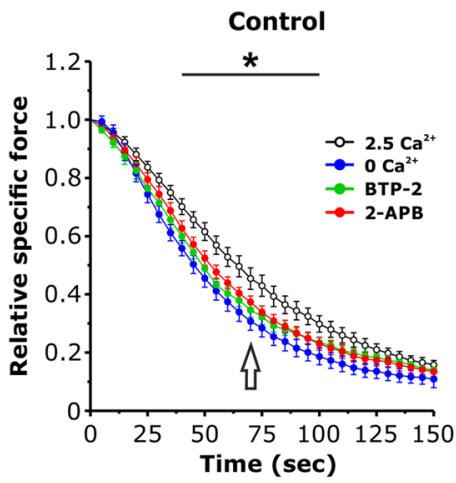

D

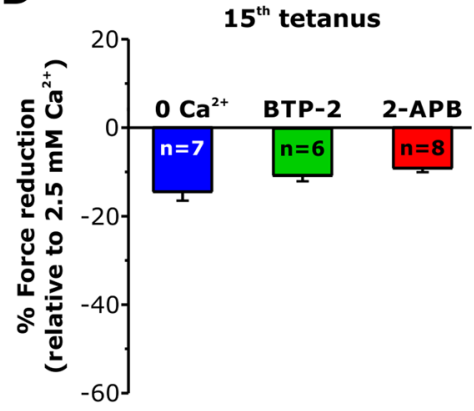

E

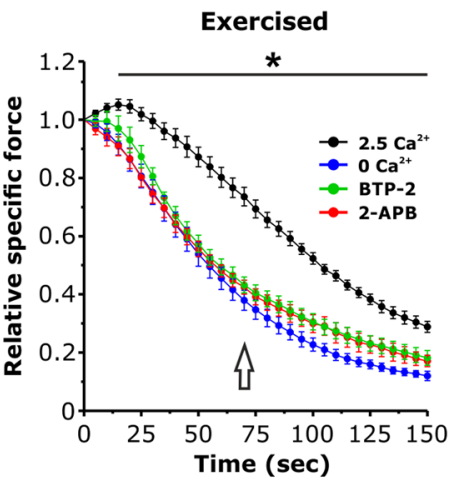

$\mathbf{F}$

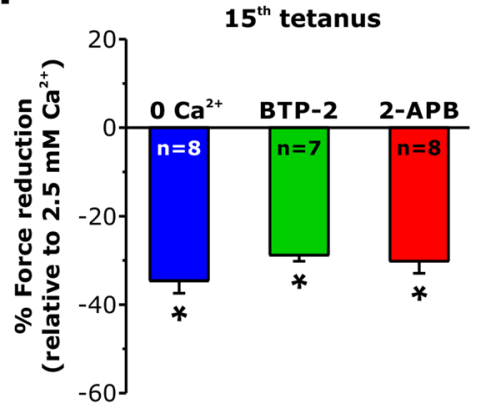

Figure 5. EDL muscles from exercised mice exhibit an enhanced resistant to fatigue that depends of $\mathrm{Ca}^{2+}$ entry. (A) Time course of average relative force decay during 30 consecutive frequency stimulus trains ( $1 \mathrm{~s}-60$ $\mathrm{Hz}$ every $5 \mathrm{~s}$ ), normalized to the $1^{\text {st }}$ stimulus train, in EDL muscles from control and exercised WT mice using a $\mathrm{KH}$ solution containing $2.5 \mathrm{mM} \mathrm{Ca}^{2+}$. (B) Bar plot showing the average fold increase in force produced by EDL muscles from exercised mice recorded during the $15^{\text {th }}$ stimulus train (arrow in $\mathbf{A}$ ) normalized to that of control muscles. (C and E) Time courses of average relative force decay during repetitive stimulation of EDL muscles from control and exercised mice in the presence of $2.5 \mathrm{mM} \mathrm{Ca}^{2+}$, in $\mathrm{Ca}^{2+}$-free $\mathrm{KH}$ solution, or presence of either $10 \mu \mathrm{M}$ BTP-2 or $100 \mu \mathrm{M}$ 2-APB. (D and F) Bar plots summarizing average force reduction during the $15^{\text {th }}$ stimulus train in the presence of $2.5 \mathrm{mM} \mathrm{Ca}^{2+}$, in $\mathrm{Ca}^{2+}$-free $\mathrm{KH}$ solution, or presence of either $10 \mu \mathrm{M} \mathrm{BTP}-2$ or $100 \mu \mathrm{M} 2$-APB. Data are shown as mean \pm SEM; ${ }^{*} \mathrm{p}<0.05$. Number of experiments $(\mathrm{n})$ reflect the number of EDL muscles analyzed for each condition.

from control mice (Fig. 5; Figs S6C and D; and S7). Moreover, this difference in fatigue susceptibility does not reflect a change in the force-frequency relationship, is observed at multiple frequencies of stimulation, and is markedly reduced by experimental interventions that reduce SOCE (e.g. removal of extracellular $\mathrm{Ca}^{2+}$ or addition of SOCE inhibitors ${ }^{36,37}$; Fig. 5 and Fig. S7).

Main events leading to formation of new SR-TT junctions at the I band. In non-muscle cells, store depletion triggers the formation of STIM1 aggregates within the ER, that then translocate to the surface membrane to trap Orail channels in STIM1-Orail puncta ${ }^{8,11}$. The findings of the present paper suggest that alternative mechanisms may coordinate STIM1-Orail assembly in skeletal muscle as, in contrast to non-excitable cells, the external membrane containing Orail proteins in skeletal muscle fibers (i.e. TTs) moves toward sites of STIM1 localization (within the I band). A model depicting the main events that occur during exercise-dependent formation of new SR-TT junctions within the I band is proposed in Fig. 6:

Figure 6A,B: fusion of free-SR (yellow) into larger tubules (Fig. 1H) and elongation of TTs (white) toward the I band (Fig. 2; and Fig. S3).

Figure 6B,C: flattening of SR membranes into parallel cisternae and docking to elongated TTs to form junctions that are connected to, but distinct from, the triad (Fig. 1; and Figs S1-S3).

In Fig. 6, while STIM1 (shown in red) is present throughout the I band both in control and exercised muscle (Fig. 6A), the latter is characterized by a remodeling of the SR (Fig. 6C). While primarily localized within TTs at the triad under control conditions (Fig. 6A), Orail (shown in green) translocates toward the Z-line following exercise, which likely results from an elongation of the TT into SR membrane stacks located deeper within the I band (Fig. 6C). The model presented in Fig. 6 also accounts for the following additional details:

STIM1 is localized both at the SR-TT and SR-SR interface. Indeed, similar electron-dense junctional strands were described previously in cells that overexpress only STIM $1^{35,42}$, consistent with the idea that ER-ER stacks containing STIM1 molecules may form independently of the presence of Orai $1^{43}$ and/or ER depletion (a more detailed Discussion of this issue in provided in Supplementary Information, Discussion: STIM1 may be present at the SR-TT and SR-SR interface). 


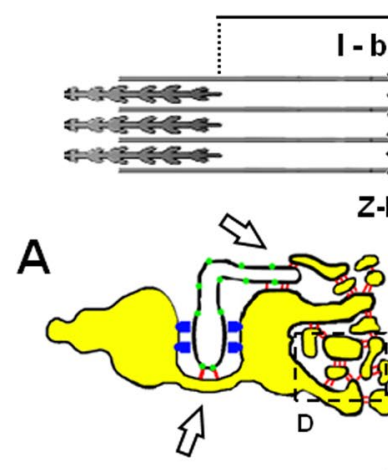

Z-1ine

B

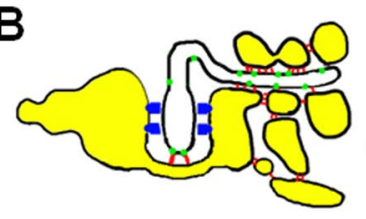

1. fusion of $\mathrm{SR}+\mathrm{T}$-tubule elongation

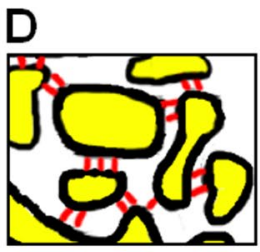

E

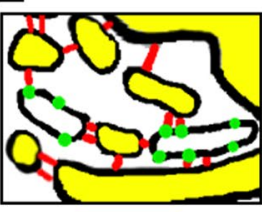

RyR1

Orai1

STIM1
CASQ1

SERCA

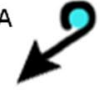

$\square$ Transverse Tubule

Sarcoplasmic Reticulum

$\mathbf{F}$

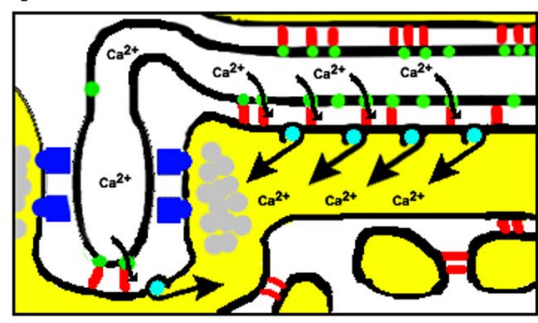

Figure 6. Model for exercise-dependent formation of Calcium Entry Units (CEUs). Main events leading to CEU formation: (A-to-B) fusion of free-SR into larger vesicles and elongation of TTs toward the I band; (B-to-C) flattening of SR membranes into parallel cisternae and final docking to elongated TTs. (D and E) Enlargements of dashed boxes in A: under control conditions, some membranes within the I band may (E) or may not (D) be of TT origin (and contain Orai1). (F) Enlargement of dashed box in C with modeling of the $\mathrm{Ca}^{2+}$ entry pathway that would allow flow-back of ions to triads during exercise/fatigue. Empty arrows in $\mathrm{A}$ point to SR-TT contacts in proximity of triads, as imaged by EM in Fig. S8.

The existence of contacts between the SR and TT in close proximity to the triad could enable STIM1-Orail interactions under control conditions (empty arrows in Fig. 6A), a scenario supported by EM images presented in Fig. S8 (a detailed Discussion of this issue is provided below).

Some of the membranes within the I band observed under control conditions could be of TT origin and contain Orail proteins (Fig. 6A-right and enlargement in Fig. 6E), which would explain low-level Orail staining within the I band under resting conditions as observed in both immunofluorescence (Fig. 3B-inset) and immunogold (Fig. 4-bottom) experiments. However, the precise molecular mechanisms that control exercise-dependent remodeling of SR and TT membranes are currently unknown.

Are the new SR-TT junctions involved in $\mathrm{Ca}^{2+}$ entry? Using constitutive and inducible muscle-specific Orail knockout mice, Carrell et al. ${ }^{18}$ concluded that: a) acute Orail-mediated $\mathrm{Ca}^{2+}$ influx plays a marginal role in limiting muscle fatigue in control (non-exercised) muscles; and b) Orail enhances muscle endurance primarily by promoting fatigue-resistant type I fiber content rather than via SOCE. Consistent with this conclusion, we found that co-localization of STIM1 and Orail is low in non-exercised muscles (see Figs 3 and 4; and Fig. S4) and contractile decline during repetitive high frequency stimulation of EDL muscle from sedentary mice is only minimally affected (Fig. 5C,D) by interventions that inhibit $\mathrm{Ca}^{2+}$ entry, e.g. $0 \mathrm{Ca}^{2+}$ or the addition SOCE inhibitors BTP-2 and 2-APB ${ }^{36,37}$. Given the absence of an effect of Orail knockout in sedentary adult mice on EDL muscle fatigue during repetitive high frequency stimulation ${ }^{18}$, the small effect of $\mathrm{Ca}^{2+}$ entry on muscle fatigue in control muscles observed in Fig. 5C,D could be mediated by a compensatory increase in another store-operated $\mathrm{Ca}^{2+}$ channels (i.e. TRPC channels) in the knockout mice. In any event, we found that a single bout of treadmill exercise caused both the formation of new SR-TT contacts in the I band that significantly increases STIM1 and Orail co-localization and reduced fatigue during repetitive high frequency stimulation (Figs 3 and 4; for additional details see Supplementary Information Discussion: Experimental evidence demonstrating the presence of both STIM1 and Orail in newly formed SR-TT junctions). However, it is important to point out that, while increased co-localization in immunofluorescence and immunogold studies provide information about the proximity between the two proteins, this does not necessarily imply a direct interaction. The formation of SR-TT stacks following exercise results in EDL muscles that are more resistant to fatigue (Fig. 5F) than EDL muscles from control mice, a difference that is essentially abolished by experimental interventions that reduce Orail-dependent SOCE (e.g. $0 \mathrm{Ca}^{2+}$ or the addition SOCE inhibitors BTP-2 and 2-APB; Fig. S7). However, as these interventions (i.e. 0 
$\mathrm{Ca}^{2+}$, BTP-2, and 2-APB) would inhibit multiple forms of $\mathrm{Ca}^{2+}$ entry, we cannot exclude the possibility that newly formed junctions also contain other types of $\mathrm{Ca}^{2+}$ entry channels other than Orail (i.e., TRPC ${ }^{44}$ ).

Nevertheless, we propose that: a) Orail-mediated $\mathrm{Ca}^{2+}$ influx into the muscle following exercise limits fatigue and that this is achieved by forming new SR-TT junctions that promote STIM1-Orail coupling (Figs 1 and S1); and b) the new SR-TT junctions formed following exercise function as Calcium Entry Units (CEUs) during repetitive muscle activity by providing structural elements to coordinate STIM1-Orail coupling and a preferential pathway to refill the SR during prolonged muscle activity (Fig. 6F). As the SR at the I band is highly-enriched in sarco-endoplasmic reticulum $\mathrm{Ca}^{2+}$ ATPases (SERCA) ${ }^{45}$, in principle the entry of extracellular $\mathrm{Ca}^{2+}$ could be efficiently re-sequestered by these pumps, while limiting diffusion of $\mathrm{Ca}^{2+}$ ions to the contractile elements (Fig. 6F). The exact contribution of SR-TT contacts in close proximity of triads (such as those shown in Fig. S8 and pointed by empty arrows in Fig. 6A) to $\mathrm{Ca}^{2+}$ entry at rest and during exercise remains to be determined. However, the combination of: i) a 5 -fold increase in CEU frequency (Fig. $1 \mathrm{H}$ ) relative to CRU frequency (resulting in a reduction in CRU/CEU ratio from 37:1 to 7:1; Table 1); and ii) increase CEU size with exercise (Fig. S2) suggest that the relative contribution of SOCE at the triad would decrease compared to that occurring via CEUs following exercise.

Relationship of present findings to prior studies in muscle. Stacks of flat-cisternae virtually identical to those displayed in Fig. 1 and Fig. S1 were previously observed in muscle from transgenic mice with junctophilin knockdown (referred to as modified-triads ${ }^{46}$ ), in CASQ1 and CASQ2 knockout ${ }^{4748}$ mice, and in triadin-junctin double knockout mice (described as SR-stacks ${ }^{49}$ ). Here we show that these structures also contain TT membranes, the molecular components for SOCE (STIM1 and Orai1, but not RyR1), exhibit a significantly smaller junctional gap than that of the triad (Fig. 1G), increase in number and size following exercise, and thus, constitute a newly-identified structural unit or organelle in skeletal muscle.

Prior studies have demonstrated that SOCE in skeletal muscle involves STIM1-Orail coupling ${ }^{15,17,50}$ and can be rapidly activated upon store depletion ${ }^{13,14,23,32}$. As our results indicate that formation of CEUs during exercise requires a relatively long time to assemble, it is unlikely that newly formed CEUs underlies rapid SOCE activation ${ }^{13,32}$. Two observations of the present study help to reconcile this apparent discrepancy: 1) preformed CEUs are present in muscle fibers under control (i.e. non-exercised) conditions, although they occur at a lower frequency and are smaller in size (Fig. 1; and Fig. S2); and 2) small electron-dense strands connecting terminal SR membranes with the lateral side of TTs are visible in close proximity of some triads (see Fig. S8; empty arrows in Fig. 6A). In fact, since the CRUs vastly out-number CEUs (37:1) under non-exercised control conditions, even a small density of STIM1-Orail co-localization within these lateral triad contacts (see Fig. S8 and Fig. 6A, arrows) could significantly contribute to rapid SOCE activation ${ }^{13,14}$ even in the absence of exercise-dependent membrane rearrangements.

Final remarks. Impaired muscle function and/or increased fatigability contributes to a reduction in the quality of life of many individuals. In this context, activity-dependent formation of junctions containing STIM1 and Orail proteins used to facilitate $\mathrm{Ca}^{2+}$ entry and $\mathrm{SR} \mathrm{Ca}^{2+}$ store replenishment may represent one beneficial component of muscle adaptation during fatigue. As altered SOCE ${ }^{41}$ and mutations in STIM1 and Orail contribute to muscle dysfunction in ageing and myopathies ${ }^{18,19,22,23,26,41}$, future investigations of CEU formation and function may lead to an improved the understanding of these pathophysiological conditions.

\section{Materials and Methods}

All experiments were conducted in male mice housed in microisolator cages under identical conditions. Mice were either sacrificed before (control) or after being subjected to a single bout of treadmill running (exercised).

Animals. $\mathrm{C} 57 \mathrm{bl} / 6$ mice were housed in microisolator cages at $20^{\circ} \mathrm{C}$ in a $12-\mathrm{h}$ light/dark cycle and provided free access to standard chow and water. Four-month old male C57bl/6 mice were randomly assigned to two experimental groups: control and exercised (mice exposed to a single bout of exercise protocol; see below). All experiments were conducted according to the Directive of the European Union 2010/63/UE and were approved by the Animal Ethical Committees of the University of Chieti. All surgeries were made to minimize animal suffering, i.e., animals were sacrificed by cervical dislocation as approved by the local University Committee on Animal Resources (15/2011/CEISA/COM).

Treadmill exercise protocol. Experiments were performed at room temperature using a running treadmill (Columbus Instruments). A first step of warm-up at low speed ( $10 \mathrm{~min}$ at $5 \mathrm{~meters} / \mathrm{min}$ ) was used to familiarize the mice with the apparatus and task. The experimental exercise protocol started immediately after the warm-up session and was designed as follows: at the beginning of the protocol, the speed was set to $10 \mathrm{~m} / \mathrm{min}$ for $25 \mathrm{~min}$ utes, then to $15 \mathrm{~m} / \mathrm{min}$ for 20 minutes, then to $20 \mathrm{~m} / \mathrm{min}$ for 15 minutes, and finally the speed was increased for $1 \mathrm{~m} / \mathrm{min}$ every 1 minute until the final speed of $25 \mathrm{~m} / \mathrm{min}$ was reached (and kept for maximum 1 minute). The protocol was stopped when mice reached exhaustion, evaluated as the inability of the animal to maintain running speed and contact with the treadmill. Variability between different mice was limited: some mice stopped just before or during the last 5 minutes of the protocol (when the speed was increased each minute by $1 \mathrm{~m} / \mathrm{min}$ ), while other mice were able to continue running until the end of the protocol.

Electron Microscopy (EM). EDL muscles were dissected from sacrificed animals, pinned on a Sylgard dish, fixed at room temperature with $3.5 \%$ glutaraldehyde in $0.1 \mathrm{M}$ sodium cacodylate ( $\mathrm{NaCaCo}$ ) buffer ( $\mathrm{pH} 7.2)$, and then stored in the fixative at $4^{\circ} \mathrm{C}$. Small bundles of fixed muscle were then post-fixed, embedded, stained en-block, and sectioned for EM, as described previously ${ }^{51}$. For TT staining, specimens were post-fixed in a mixture of $2 \% \mathrm{OsO}_{4}$ and $0.8 \% \mathrm{~K}_{3} \mathrm{Fe}(\mathrm{CN})_{6}$ for $1-2 \mathrm{~h}$ followed by a rinse with $0.1 \mathrm{M} \mathrm{NaCaCo}$ buffer with $75 \mathrm{mM} \mathrm{CaCl}_{2}$. 
Ultrathin sections ( $~ 50 \mathrm{~nm})$ were cut using a Leica Ultracut R microtome (Leica Microsystem) with a Diatome diamond knife (Diatome Ltd.) and double-stained with uranyl acetate and lead citrate. Sections were viewed in a FP 505 Morgagni Series 268D electron microscope (FEI Company), equipped with Megaview III digital camera and Soft Imaging Systemat $60 \mathrm{kV}$ or $100 \mathrm{kV}$ (for TT staining preparations).

Immunogold labeling for EM. EDL muscles were fixed for $20 \mathrm{~min}$ at room temperature in a fixative mix containing $2 \%$ paraformaldehyde and $0.5 \%$ glutaraldehyde (in PBS buffer). Small bundles of fixed fibers were blocked for 1 hour in PBS containing 1\% bovine serum albumin (BSA), 10\% goat serum, and permeabilized with $0.5 \%$ TritonX-100. After incubation with primary antibodies, secondary antibodies conjugated with Nanogold ${ }^{\circledR}$ particles (either goat anti-mouse or goat anti-rabbit; Nanoprobes) were applied for $2 \mathrm{~h}$ at $4{ }^{\circ} \mathrm{C}(1: 100)$. Samples were then post-fixed in $1 \%$ glutaraldehyde PBS buffer at room temperature and incubated with reagents to enhance the signal (Goldenhance ${ }^{\mathrm{TM}}$ EM Formulation, Nanoprobes) for 5 minutes. Fiber bundles were finally post-fixed, embedded, and sectioned with standard EM protocols. Primary antibodies used: mouse monoclonal anti-RyR1/RyR3 34 C antibody, (1:30, Developmental Studies Hybridoma Bank); rabbit polyclonal anti-STIM1, (1:100, Sigma Aldrich); rabbit polyclonal anti-Orail, (1:20, Thermo Scientific).

\section{Quantitative analysis of EM and immunogold preparations.}

a) The percentage of fibers exhibiting stacks of flat-long cisternae/tubes and the number of stacks per $100 \mu \mathrm{m}^{2}$ area/section (data in Fig. 1E,F) were determined from electron micrographs of non-overlapping regions randomly collected from transverse sections. In each specimen, 9-21 cross-sectional fibers were analyzed and in each fiber 5 micrographs were taken at 28,000x magnification.

b) The junctional gap in CRUs (or triads), between SR vesicles (in control) and in stacks of flat-cisternae in electron micrographs were taken at 44,000x or 56,000x magnification (data in Fig. 1G).

c) The average size of SR vesicles in proximity of stacks (data in Fig. $1 \mathrm{H}$ ) was measured in transverse sections from electron micrographs at 44,000x magnification.

d) The extension of non-triadic TT network within at the I band (data in Fig. 2F) was measured in electron micrographs at 28,000x magnification of non-overlapping regions randomly collected from transverse sections of samples stained with ferrocyanide (see also Fig. S3). In each specimen, 9-21 cross-sectioned fibers were analyzed and in each fiber 5 micrographs were taken.

e) The average length of the membrane-membrane contacts in the stacks of flat-cisternae was measured in micrographs taken at 56,000x magnification as shown in Fig. S2C (labeled by cyan dots).

f) The number of membrane elements contained in the stacks of flat-cisternae (data in Fig. S2D) was obtained in micrographs taken at $28.000 x$ and $44,000 x$ magnification by counting single elements clearly delimited by membrane phospholipids.

g) The number of CRUs and CEUs in $100 \mu \mathrm{m}^{2}$ of EM section (data in Table 1) was determined from electron micrographs of non-overlapping regions randomly collected from longitudinal sections. In each specimen, 7 fibers were analyzed, and in each fiber, 5-10 micrographs were taken at 18,000x magnification.

h) The distance of gold particles from the nearest $Z$ line in longitudinal sections was measured in immunogold labeled EM sections (data in Fig. 4; and Fig. S4). Quantitative data from electron micrographs in sections b), c), d), e), and h) (above) were obtained using the Analy-SIS software of the EM digital camera (Olympus Soft Imaging Solutions).

Immunofluorescence labeling and confocal microscopy (CM). EDL muscles were fixed and processed for immunofluorescence as previously described ${ }^{51}$. Primary antibodies used: a) mouse monoclonal anti-RyR1/RyR3 (34 C antibody, 1:30, Developmental Studies Hybridoma Bank); b) rabbit polyclonal anti-STIM1 (1:100, Sigma Aldrich); c) mouse monoclonal anti-STIM1 (1:50, BD Biosciences); or d) rabbit polyclonal anti-Orai1, (1:20, Thermo Scientific). Secondary antibodies used: a) Cy5-labeled goat anti-mouse IgG (1:50); or b) Cy3-labeled goat anti-rabbit (1:200). Confocal images were acquired using a Zeiss LSM510 META system equipped with a Zeiss Axiovert 200 inverted microscope and a Plan Neofluar oil-immersion objective (100X/1.3 NA). Fluorescence image profiles were obtained from LSM 3.0 image analysis software by Zeiss. Co-localization was determined by Imaris 7.2.3 software (Bitplane) and quantified by an evaluation of the Pearson's correlation coefficient values.

Ex-vivo fatigue protocol. Intact EDL muscles were removed from hind limbs of control and exercised mice and placed in a dish with a Krebs-Henseleit $(\mathrm{KH})$ solution containing: $118 \mathrm{mM} \mathrm{NaCl} ; 5 \mathrm{mM} \mathrm{KCl} ; 2.5 \mathrm{mM} \mathrm{CaCl}$; $1 \mathrm{mM} \mathrm{KH}_{2} \mathrm{PO}_{4} ; 1 \mathrm{mM} \mathrm{MgSO}_{4} ; 25 \mathrm{mM} \mathrm{NaHCO}_{3}$; and $11 \mathrm{mM}$ Glucose; $\mathrm{pH}$ 7.4. Muscles were pinned, tied with fine silk sutures at each end, and mounted vertically between two platinum electrodes immersed in an organ chamber filled with $\mathrm{KH}$ solution ${ }^{52}$ and attached to a servo motor and force transducer (1200 A, Aurora Scientific). Before starting the experimental protocol, stimulation level and optimal muscle length $\left(L_{0}\right)$ were determined using a series of $80 \mathrm{~Hz}$ stimulation trains in order to stretch the muscle to the length that generated maximal force $\left(F_{0}\right)$. Twitch and tetanic contractile properties, as well as force-frequency parameters, were then measured. Following these baseline measurements, EDL muscles were then subjected to a repetitive stimulation fatigue protocol consisting of 30 consecutive stimulus trains at $60 \mathrm{~Hz}$ of frequency (each pulse having a duration of $1 \mathrm{~s}$ ) applied every $5 \mathrm{~s}$ while being continuously perfused with $\mathrm{KH}$ solution. To assess the relative contribution of extracellular $\mathrm{Ca}^{2+}$ entry, some experiments were conducted either in the absence of external $\mathrm{Ca}^{2+}$ (equimolar substitution with $\mathrm{Mg}^{2+}$ ) or in presence of $10 \mu \mathrm{M}$ BTP-2 or $100 \mu \mathrm{M} 2-\mathrm{APB}$, two established inhibitors of SOCE ${ }^{36-39}$. In a separate set of experiments, EDL muscles from control and exercised mice perfused with control KH solution were subjected 
to a similar repetitive stimulation protocol, but using a simulation frequency $(100 \mathrm{~Hz})$ at the peak of the force frequency curve (i.e. 30 consecutive $1 \mathrm{~s}$-stimulus trains at $100 \mathrm{~Hz}$ of frequency applied every $5 \mathrm{~s}$ ). Specific force $\left(\mathrm{mN} / \mathrm{mm}^{2}\right)$ was calculated by normalizing the absolute force $(\mathrm{mN})$ to the physiological cross sectional area (in $\left.\mathrm{mm}^{2}\right)$ obtained as follows: wet weight $(\mathrm{mg}) /\left(L_{0}(\mathrm{~mm}) \times 1.06\left(\mathrm{mg} / \mathrm{mm}^{3}\right) \times 0.44\right)$. All experiments were carried out at room temperature.

Statistical analyses. Statistical significance in all experiments was evaluated using a two-tailed unpaired Student's t test for the $95 \%$ confidence intervals, with the exception of statistical significance in Figs 4 and S2F (determined using a two-tailed Fisher's exact test) and the ex vivo muscle fatigue results of Figs 5, S6 and S7 (evaluated using a one-way ANOVA followed by post-hoc Tuckey test for multiple comparisons). All data were presented as mean \pm SEM.

\section{References}

1. Putney, J. W. Jr. A model for receptor-regulated calcium entry. Cell Calcium 7, 1-12 (1986)

2. Parekh, A. B. \& Penner, R. Store depletion and calcium influx. Physiol Rev 77, 901-930 (1997)

3. Roos, J. et al. STIM1, an essential and conserved component of store-operated $\mathrm{Ca}^{2+}$ channel function. J Cell Biol 169, 435-445 (2005).

4. Liou, J. et al. STIM is a Ca ${ }^{2+}$ sensor essential for $\mathrm{Ca}^{2+}$ store-depletion-triggered $\mathrm{Ca}^{2+}$ influx. Curr Biol 15, 1235-1241 (2005).

5. Zhang, S. L. et al. STIM1 is a $\mathrm{Ca}^{2+}$ sensor that activates CRAC channels and migrates from the $\mathrm{Ca}^{2+}$ store to the plasma membrane. Nature 437, 902-905 (2005).

6. Feske, S. et al. A mutation in Orail causes immune deficiency by abrogating CRAC channel function. Nature 441, 179-185 (2006).

7. Vig, M. et al. CRACM1 is a plasma membrane protein essential for store-operated $\mathrm{Ca}^{2+}$ entry. Science 312, 1220-1223 (2006).

8. Wu, M. M., Buchanan, J., Luik, R. M. \& Lewis, R. S. Ca ${ }^{2+}$ store depletion causes STIM1 to accumulate in ER regions closely associated with the plasma membrane. J Cell Biol 174, 803-813 (2006).

9. Luik, R. M., Wang, B., Prakriya, M., Wu, M. M. \& Lewis, R. S. Oligomerization of STIM1 couples ER calcium depletion to CRAC channel activation. Nature 454, 538-542 (2008).

10. Liou, J., Fivaz, M., Inoue, T. \& Meyer, T. Live-cell imaging reveals sequential oligomerization and local plasma membrane targeting of stromal interaction molecule 1 after $\mathrm{Ca}^{2+}$ store depletion. Proc Natl Acad Sci USA 104, 9301-9306 (2007).

11. Lewis, R. S. The molecular choreography of a store-operated calcium channel. Nature 446, 284-287 (2007).

12. Kurebayashi, N. \& Ogawa, Y. Depletion of $\mathrm{Ca}^{2+}$ in the sarcoplasmic reticulum stimulates $\mathrm{Ca}^{2+}$ entry into mouse skeletal muscle fibres. J Physiol 533, 185-199 (2001).

13. Launikonis, B. S. \& Rios, E. Store-operated $\mathrm{Ca}^{2+}$ entry during intracellular $\mathrm{Ca}^{2+}$ release in mammalian skeletal muscle. J Physiol 583, 81-97 (2007)

14. Launikonis, B. S., Barnes, M. \& Stephenson, D. G. Identification of the coupling between skeletal muscle store-operated Ca ${ }^{2+}$ entry and the inositol trisphosphate receptor. Proc Natl Acad Sci USA 100, 2941-2944 (2003).

15. Lyfenko, A. D. \& Dirksen, R. T. Differential dependence of store-operated and excitation-coupled Ca ${ }^{2+}$ entry in skeletal muscle on STIM1 and Orai1. J Physiol 586, 4815-4824 (2008).

16. Dirksen, R. T. Checking your SOCCs and feet: the molecular mechanisms of $\mathrm{Ca}^{2+}$ entry in skeletal muscle. J Physiol 587, 3139-3147 (2009).

17. Wei-Lapierre, L., Carrell, E. M., Boncompagni, S., Protasi, F. \& Dirksen, R. T. Orail-dependent calcium entry promotes skeletal muscle growth and limits fatigue. Nat Commun 4, 2805 (2013).

18. Carrell, E. M., Coppola, A. R., McBride, H. J. \& Dirksen, R. T. Orail enhances muscle endurance by promoting fatigue-resistant type I fiber content but not through acute store-operated $\mathrm{Ca}^{2+}$ entry. FASEB J, https://doi.org/10.1096/fj.201600621R (2016).

19. Zhao, X. et al. Compromised store-operated $\mathrm{Ca}^{2+}$ entry in aged skeletal muscle. Aging Cell 7, 561-568 (2008).

20. Thornton, A. M. et al. Store-operated $\mathrm{Ca}^{2+}$ entry (SOCE) contributes to normal skeletal muscle contractility in young but not in aged skeletal muscle. Aging (Albany NY) 3, 621-634 (2011).

21. Payne, A. M., Jimenez-Moreno, R., Wang, Z. M., Messi, M. L. \& Delbono, O. Role of $\mathrm{Ca}^{2+}$, membrane excitability, and Ca ${ }^{2+}$ stores in failing muscle contraction with aging. Exp Gerontol 44, 261-273 (2009).

22. Edwards, J. N., Blackmore, D. G., Gilbert, D. F., Murphy, R. M. \& Launikonis, B. S. Store-operated calcium entry remains fully functional in aged mouse skeletal muscle despite a decline in STIM1 protein expression. Aging Cell 10, 675-685 (2011).

23. Zhao, X., Moloughney, J. G., Zhang, S., Komazaki, S. \& Weisleder, N. Orail mediates exacerbated Ca ${ }^{2+}$ entry in dystrophic skeletal muscle. PLoS One 7 (2012).

24. Goonasekera, S. A. et al. Enhanced $\mathrm{Ca}^{2+}$ influx from STIM1-Orail induces muscle pathology in mouse models of muscular dystrophy. Hum Mol Genet 23, 3706-3715 (2014).

25. Onopiuk, M. et al. Store-operated calcium entry contributes to abnormal $\mathrm{Ca}^{2+}$ signalling in dystrophic mdx mouse myoblasts. Arch Biochem Biophys 569, 1-9 (2015).

26. Pan, Z., Brotto, M. \& Ma, J. Store-operated $\mathrm{Ca}^{2+}$ entry in muscle physiology and diseases. BMB Rep 47, 69-79 (2014).

27. Bohm, J. et al. Constitutive activation of the calcium sensor STIM1 causes tubular-aggregate myopathy. Am J Hum Genet 92 , 271-278 (2013).

28. Nesin, V. et al. Activating mutations in STIM1 and ORAI1 cause overlapping syndromes of tubular myopathy and congenital miosis. Proc Natl Acad Sci USA 111, 4197-4202 (2014).

29. Endo, Y. et al. Dominant mutations in ORAI1 cause tubular aggregate myopathy with hypocalcemia via constitutive activation of store-operated $\mathrm{Ca}^{2+}$ channels. Hum Mol Genet 24, 637-648 (2015).

30. Okuma, H. et al. Tubular aggregate myopathy caused by a novel mutation in the cytoplasmic domain of STIM1. Neurol Genet 2, e50, $10.1212(2016)$

31. Garibaldi, M. et al. A novel gain-of-function mutation in ORAI1 causes late-onset tubular aggregate myopathy and congenital miosis. Clin Genet, https://doi.org/10.1111/cge.12888 (2016).

32. Edwards, J. N. et al. Ultra-rapid activation and deactivation of store-operated $\mathrm{Ca}(2+)$ entry in skeletal muscle. Cell Calcium 47 , 458-467 (2010).

33. Schneider, M. F. Control of calcium release in functioning skeletal muscle fibers. Annu Rev Physiol 56, 463-484 (1994).

34. Franzini-Armstrong, C., Protasi, F. \& Ramesh, V. Shape, size, and distribution of $\mathrm{Ca}^{2+}$ release units and couplons in skeletal and cardiac muscles. Biophysical Journal 77, 1528-1539 (1999).

35. Orci, L. et al. From the Cover: STIM1-induced precortical and cortical subdomains of the endoplasmic reticulum. Proc Natl Acad Sci USA 106, 19358-19362 (2009).

36. Zitt, C. et al. Potent inhibition of $\mathrm{Ca} 2+$ release-activated $\mathrm{Ca}^{2+}$ channels and T-lymphocyte activation by the pyrazole derivative BTP2. J Biol Chem 279, 12427-12437 (2004).

37. Bootman, M. D. et al. 2-aminoethoxydiphenyl borate (2-APB) is a reliable blocker of store-operated $\mathrm{Ca}^{2+}$ entry but an inconsistent inhibitor of InsP3-induced $\mathrm{Ca}^{2+}$ release. FASEB J 16, 1145-1150 (2002) 
38. Hendron, E. et al. Potent functional uncoupling between STIM1 and Orail by dimeric 2 -aminodiphenyl borinate analogs. Cell Calcium 56, 482-492 (2014).

39. Ohga, K., Takezawa, R., Arakida, Y., Shimizu, Y. \& Ishikawa, J. Characterization of YM-58483/BTP2, a novel store-operated Ca ${ }^{2+}$ entry blocker, on T cell-mediated immune responses in vivo. Int Immunopharmacol 8, 1787-1792 (2008).

40. Zhao, X. et al. Enhanced resistance to fatigue and altered calcium handling properties of sarcalumenin knockout mice. Physiol Genomics 23, 72-78 (2005).

41. Brotto, M. Aging, sarcopenia and store-operated calcium entry: a common link? Cell Cycle 10, 4201-4202 (2011).

42. Perni, S., Dynes, J. L., Yeromin, A. V., Cahalan, M. D. \& Franzini-Armstrong, C. Nanoscale patterning of STIM1 and Orai1 during store-operated $\mathrm{Ca}^{2+}$ entry. Proc Natl Acad Sci USA 112, E5533-5542 (2015).

43. Park, C. Y. et al. STIM1 clusters and activates CRAC channels via direct binding of a cytosolic domain to Orai1. Cell 136, 876-890 (2009).

44. He, L. P., Hewavitharana, T., Soboloff, J., Spassova, M. A. \& Gill, D. L. A functional link between store-operated and TRPC channels revealed by the 3,5-bis(trifluoromethyl)pyrazole derivative, BTP2. J Biol Chem 280, 10997-11006 (2005).

45. Franzini-Armstrong, C. \& Ferguson, D. G. Density and disposition of Ca2 +-ATPase in sarcoplasmic reticulum membrane as determined by shadowing techniques. Biophys J 48, 607-615 (1985).

46. Ko, J. K. et al. A versatile single-plasmid system for tissue-specific and inducible control of gene expression in transgenic mice. FASEB J 25, 2638-2649 (2011).

47. Boncompagni, S., Protasi, F. \& Franzini-Armstrong, C. Sequential stages in the age-dependent gradual formation and accumulation of tubular aggregates in fast twitch muscle fibers: SERCA and calsequestrin involvement. Age (Dordr) 34, 27-41 (2012).

48. Valle, G. et al. Characterization of fast-twitch and slow-twitch skeletal muscles of calsequestrin 2 (CASQ2)-knock out mice: unexpected adaptive changes of fast-twitch muscles only. J Muscle Res cell Motil 37, 225-233 (2016).

49. Boncompagni, S. et al. Triadin/Junctin double null mouse reveals a differential role for Triadin and Junctin in anchoring CASQ to the jSR and regulating $\mathrm{Ca}(2+)$ homeostasis. PLoS One 7, e39962 (2012).

50. Stiber, J. et al. STIM1 signalling controls store-operated calcium entry required for development and contractile function in skeletal muscle. Nat Cell Biol 10, 688-697 (2008).

51. Pietrangelo, L. et al. Age-dependent uncoupling of mitochondria from $\mathrm{Ca}^{2+}$ release units in skeletal muscle. Oncotarget 6 , 35358-35371 (2015).

52. Michelucci, A. et al. Antioxidants protect calsequestrin-1 knockout mice from halothane- and heat-induced sudden death. Anesthesiology 123, 603-617 (2015).

\section{Acknowledgements}

This study was supported by the following grants: a) Italian Telethon ONLUS Foundation (Rome, Italy): GGP13213 to FP; Italian Ministry of Health (Rome, Italy): GR-2011-02352681 to SB c) National Institute of Health (Bethesda, MD, U.S.A): AR059646 to RTD (and subcontract to FP); d) Muscular Dystrophy Association (MDA275574) to RTD (and subcontract to FP). We thank Aurora Fusella for technical support and assistance in the staining of samples for immunogold EM.

\section{Author Contributions}

F.P., S.B. and R.T.D. conceived and directed the study. S.B., A.M. and L.P. performed the experimental work and data analysis. In detail, S.B. and L.P. performed imaging and quantitative data analysis of electron microscopy; A.M. performed confocal imaging and functional analysis. Finally, F.P., R.T.D., S.B., and A.M. wrote the manuscript.

\section{Additional Information}

Supplementary information accompanies this paper at https://doi.org/10.1038/s41598-017-14134-0.

Competing Interests: The authors declare that they have no competing interests.

Publisher's note: Springer Nature remains neutral with regard to jurisdictional claims in published maps and institutional affiliations.

(c) (i) Open Access This article is licensed under a Creative Commons Attribution 4.0 International

License, which permits use, sharing, adaptation, distribution and reproduction in any medium or format, as long as you give appropriate credit to the original author(s) and the source, provide a link to the Creative Commons license, and indicate if changes were made. The images or other third party material in this article are included in the article's Creative Commons license, unless indicated otherwise in a credit line to the material. If material is not included in the article's Creative Commons license and your intended use is not permitted by statutory regulation or exceeds the permitted use, you will need to obtain permission directly from the copyright holder. To view a copy of this license, visit http://creativecommons.org/licenses/by/4.0/.

(C) The Author(s) 2017 